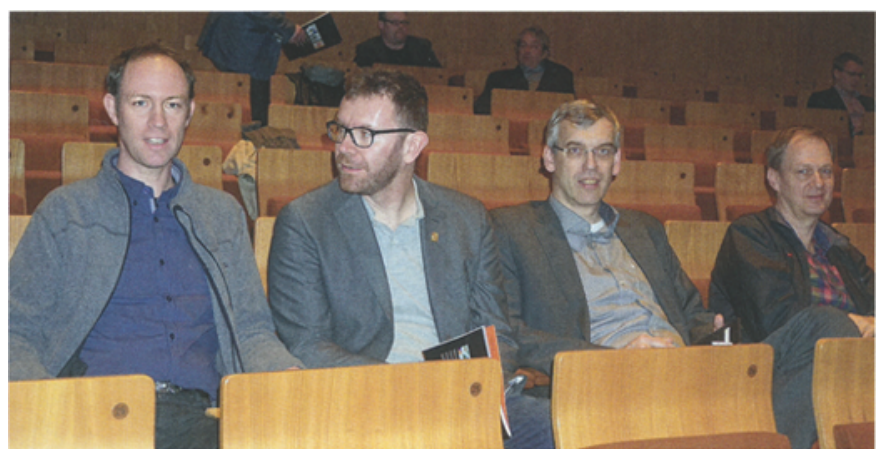

Abb. 2: Vier Mitglieder aus dem Vorstand der DGfK beim Neujahrsempfang. Von links: Steffen Hild (Sekretär), Peter Neumann (Schatzmeister), Prof. Dr. Jochen Schiewe (Vizepräsident National), Dr. Thomas Chudy (Sprecher der Sektionen) Foto: Mark Vetter

aus Wirtschaft und Hochschulen haben das Symposium zu einer spannenden Vortragsreihe gemacht: Begonnen hat Prof. Dr. Gerd Buziek (Esri) mit visionären Ansichten zum Thema „Digitale Transformationen - Mit GIS die Zukunft gestalten“. Danach kam Prof. Dr. Frank Schüssler (Jade Hochschule) mit dem Thema „Forschendes Lehren im Geomarketing“" zu Wort. Er hat eine Lanze gebrochen für die wichtige

Verbindung und das große Potential, welches sich aus der Kombination aus Lehre und Forschung ergeben kann. Es folgte ein Beitrag von Walter Erlenbach (DDGI), der Beispiele aus der Praxis des Geomarketings aufgezeigt hatte. Dr. Peter Hecker von (Geokomm e. V.) führte in seinem anschließenden Vortrag weitere Aspekte des Geomarketing aus und schließlich stellte Holger Bronsch von AED Sicad, den Wert von

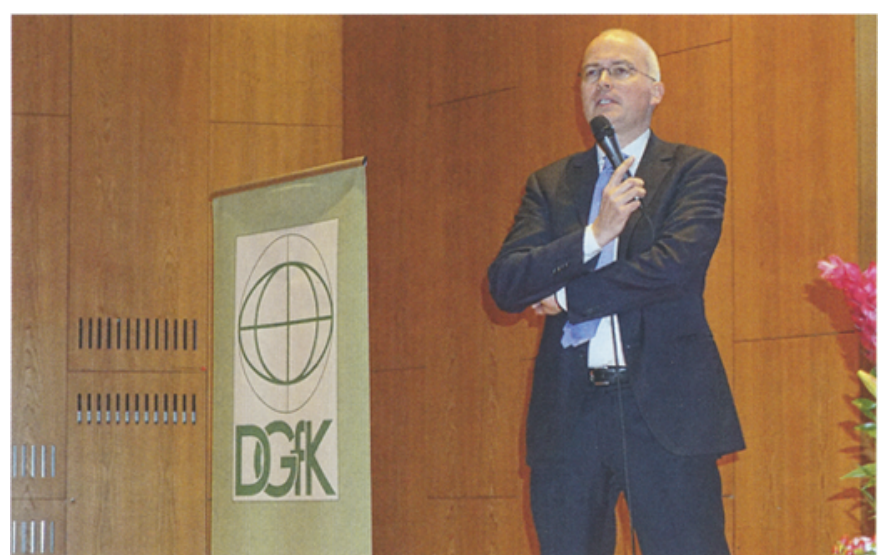

Abb. 3: Michael Bültmann, Geschäftsfüher der here $\mathrm{GmbH}$ während des Festvortrages. (Foto: Mark Vetter)

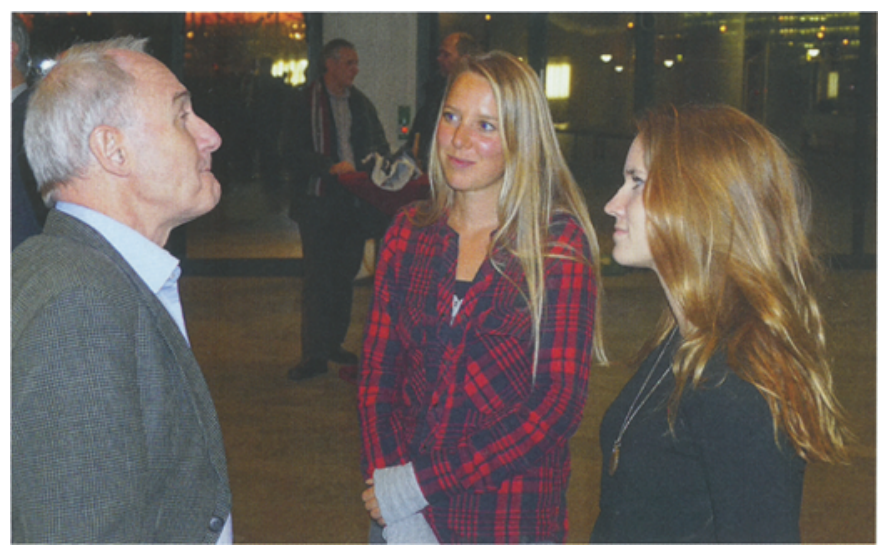

Abb. 4: Vizepräsident der DGfK, Prof. Dr. Manfred Buchroithner im Gespräch mit Geographie Master-Studentinnen beim Empfang. (Foto: Mark Vetter)
Geoinformationen am Wirtschaftsstandort Deutschland heraus. Höhepunkt nach Grußworten und Neujahrswünschen vom Sektionsleiter der DGfK BerlinBrandenburg (Horst Kremers) und natürlich vom Präsidenten der DGfK, Prof. Dr. Manfred Weissensee war ein Vortrag vom Geschäftsführer der here $\mathrm{GmbH}$ (Michael Bültmann). Er stellt dar, welche Bedeutung hochqualitative Karten(dienste) und Geodaten für den Automotive-Bereich, aber auch andere Wirtschaftsbereiche haben. Sein Beitrag war betitelt mit „Bedeutung von digitalen Karten und Location Intelligence für neue Geschäftsmodelle“. Der Neujahrsempfang der Deutschen Gesellschaft für Kartographie 2016 war ein voller Erfolg und aller Voraussicht nach, werden wir am 19.1.2017 wieder das neue Jahr in Berlin an diesem Standort begrüßen.

\section{Langjährige \\ DGfK-Mitgliedschaft März/April 2016}

Folgenden Mitgliedern dankt die Deutsche Gesellschaft für Kartographie für ihre langjährige Treue:

\section{Mitglied seit 50 Jahren:} Volker Mann, 76199 Karlsruhe

\section{Mitglied seit 40 Jahren:}

Dipl.-Des. Eva Schümann, 47803 Krefeld

Dipl.-Ing. (FH) Bernd Beckmann, 30171 Hannover

Dipl.-Ing. Marion Ewinger, 53842 Troisdorf

Dipl.-Ing. (FH) Susanne Kowalewski, 24251 Osdorf Dr.-Ing. Johannes Schoppmeyer, 53757 Sankt Augustin

Dipl.-Ing. (FH) Hartmut Wiethaup, 61476 Kronberg am Taunus

\section{Mitglied seit 25 Jahren:}

Dipl.-Ing. Wolfram Dolz, 01324 Dresden

Dipl.-Ing. Ursula Guske, 10713 Berlin
Dipl.-Ing. Irene Nöske, 13187 Berlin Dipl.-Ing. (FH) Christian Rolle, 83607 Holzkirchen Dipl.-Geogr. Axel Borchert, 14169 Berlin

\section{Geburtstage}

2.3. Prof. Dr.-Ing. Helmut Kuhn, 13403 Berlin, 65 Jahre

2.3. Prof. Dr. Uta Lindgren, 80686 München, 75 Jahre 3.3. Dipl.-Ing. Eberhard Herdeg, 70184 Stuttgart, 75 Jahre

3.3. Dipl.-Ing. Marlies Krause, 50968 Köln, 60 Jahre 5.3. Wolfgang Helbig, 04328 Leipzig, 80 Jahre 5.3. Jürgen Sieversen, 82024 Taufkirchen, 85 Jahre 13.3. Dipl.-Ing. Marion Ewinger, 53842 Troisdorf, 60 Jahre 14.3. Dr. Hubert Bischoff, 28857 Syke, 65 Jahre 17.3. Dr. phil. Volkmar Kroesch, 53129 Bonn, 75 Jahre

18.3. Dr.-Ing.

Wilfried Staufenbiel, 30880 Laatzen, 75 Jahre 21.3. Dipl.-Ing. (FH) Barbara Schultze, 60439 Frankfurt am Main, 60 Jahre

28.3. Dipl.-Ing. (FH)

Winfried Roeding, 53117 Bonn, 80 Jahre 3.4. Dipl.-Ing. Karl Andreas, 76199 Karlsruhe, 85 Jahre 5.4. Prof. Dipl.-Ing. Herbert Hufnagel, 80636 München, 85 Jahre 9.4. Dr. Karl-Joachim Mahncke, 53797 Lohmar, 85 Jahre

10.4. Prof. Dr.-Ing. Dietmar Grünreich, 30952 Ronnenberg, 70 Jahre 18.4. Manfred Steuerer, 83727 Schliersee-Neuhaus, 75 Jahre

19.4. Dipl.-Ing. Achim Hoppe, 04159 Leipzig, 80 Jahre

24.4. Rainer Schulten, 45721 Haltern, 60 Jahre 30.4. Dr.-Ing. Christian Clauß, 06126 Halle (Saale), 94 Jahre 